\title{
Left ventricular contractile reserve in stress echocardiography: the bright side of the force
}

\author{
Eugenio Picano ${ }^{1}$, Tonino Bombardini ${ }^{2}$, Tamara Kovačević Preradović ${ }^{2}$, Lauro Cortigiani ${ }^{3}$, \\ Karina Wierzbowska-Drabik ${ }^{4}$, Quirino Ciampi ${ }^{5}$ \\ ${ }^{1}$ Institute of Clinical Physiology, National Council Research, Pisa, Italy \\ ${ }^{2}$ School of Medicine, University Clinical Centre of The Republic of Srpska, Banja Luka, Bosnia and Herzegovina \\ ${ }^{3}$ Cardiology Division, San Luca Hospital, Lucca, Italy \\ ${ }^{4}$ Chair and Department of Cardiology, Medical University of Lodz, Lodz, Poland \\ ${ }^{5}$ Department of Cardiology, Fatebenefratelli Hospital of Benevento, Benevento, Italy
}

\begin{abstract}
A bstract
Stress echocardiography (SE) is based on the detection of regional wall motion abnormalities (RWMA) mirroring a physiologically critical epicardial artery stenosis which determines subendocardial underperfusion. Recently, the core protocol of SE has been enriched by the addition of left ventricular contractile reserve (LVCR) based on force. Changes in force can be caused by microvascular and/or epicardial coronary artery disease, but also by myocardial scar, necrosis, and/or sub-epicardial layer disease. Left ventricular contractile reserve is calculated as the stress-to-rest ratio of force (systolic arterial pressure measured by cuff sphygmomanometer to end-systolic volume determined by two-dimensional echocardiography). In contrast to the ejection fraction, force is not dependent on changes in preload and afterload. Cut-off values for a preserved LVCR are $>2.0$ for dobutamine or exercise stress and $>1.1$ for vasodilators, which are weaker inotropic stimuli. Patients with a "strong" heart (normal LVCR values) have a better outcome than patients with a "weak" heart (reduced LVCR values), and this is the prognostic "bright side of the force," meaning that the prognostic value of force-based contractile reserve is higher than that of ejection fraction-based contractile reserve or RWMA.

The addition of force to standard SE based on RWMA detection increases the spectrum of risk stratification without any significant increase in imaging time and only a slight increase in analysis time. In both ischaemic (with RWMA) and non-ischaemic (without RWMA) hearts, the preserved force is associated with a more benign prognosis. The prospective multicentre international Stress Echo 2020 trial which started in September 2016 has already recruited > 5000 patients with dual RWMA-force imaging and will systematically test the impact of force on the prognosis within and beyond coronary artery disease, including heart failure and hypertrophic cardiomyopathy.
\end{abstract}

Key words: end-systolic volume, force, left ventricular contractility, stress echocardiography, wall motion abnormalities

Kardiol Pol 2019; 77, 2: 164-172

\section{ADDING FORCE TO STRESS} ECHOCARDIOGRAPHY

Stress echocardiography (SE) based on regional wall motion abnormalities (RWMA) is a well-established technique for diagnosis and risk stratification in several cardiovascular conditions, within and beyond coronary artery disease (CAD) [1-5]. In recent years, the new cost-conscious and radiation-conscious climate [6-9] was the main driver of the observed relative growth of SE over competing cardiac stress imaging techniques $[10,11]$. As an example, in the Mayo Clinic of Rochester, NY, USA, the myocardial perfusion imaging/SE utilisation ratio was 10 to 0 in 1999 and 1 to 5 in 2012 [12].

In spite of the increasing use of SE based on RWMA, this technique has limitations in contemporary populations. The positivity rate for SE decreased in the last decades, from $40 \%$ to less than $10 \%$ [13-15], mostly because of a reduction in

Address for correspondence:

Eugenio Picano, MD, PhD, Institute of Clinical Physiology, National Council Research, Via Giuseppe Moruzzi 1, Pisa 56124, Italy, tel: + 39-050-315 2398,

fax: +39-050-315 2374, e-mail: picano@ifc.cnr.it

Received: 8.01.2019 Accepted: 8.01.2019 Available as AoP: 8.01.2019

Kardiologia Polska Copyright (c) Polish Cardiac Society 2019 


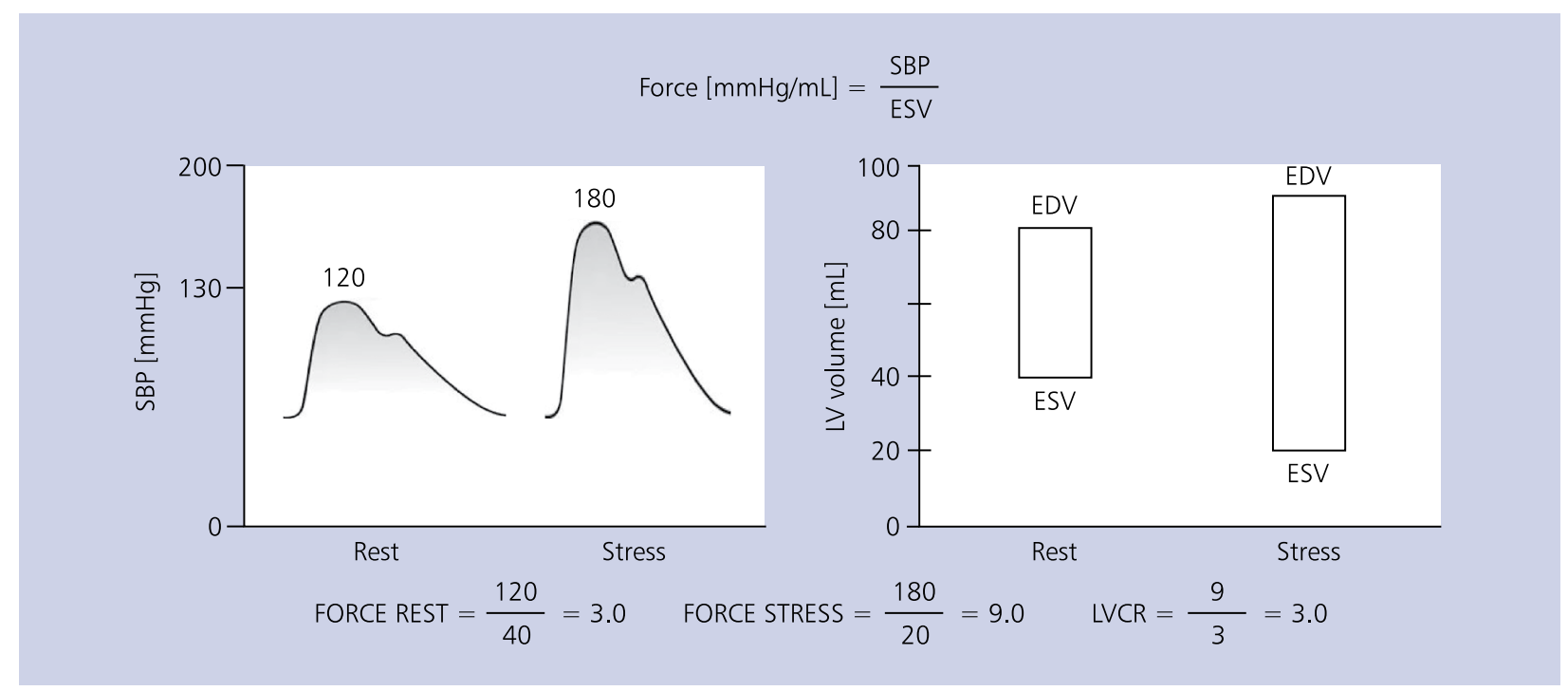

Figure 1. An example of a calculation of force and left ventricular contractile reserve (LVCR). In simplified terms, force is calculated as the ratio of peak systolic blood pressure (SBP; obtained noninvasively with cuff sphygmomanometer) to end-systolic volume (ESV) of the left ventricle (LV). LVCR is calculated as the ratio of stress to rest force; EDV — end-diastolic volume

pre-test probability of disease at referral. The predictive value of a negative test for RWMA is low, but not as low as would be desired or as low as for other competing techniques: the annual hard event rate was $1.7 \%$ in the years 2000 to 2016 [16, 17 ], while in the early series published in the 1990s, when SE was applied to more diseased populations evaluated off anti-anginal therapy, this rate was $<1 \%[18-20]$.

The awareness of these limitations was a potent stimulus for a conceptual and methodological remodelling of the technique, which led to the creation of a new standard: quadruple-imaging SE [21]. In the new version, SE simultaneously provides imaging of RWMA (a mirror of physiologically significant epicardial artery stenosis) [22, 23], extravascular lung water (with B-lines and lung ultrasound) [24-26], left ventricular contractile reserve (LVCR) based on force [27], and coronary flow velocity reserve (CFVR, targeting coronary microcirculation) [28]. This new quadruple-imaging protocol of $\mathrm{SE}$ is referred to as the $\mathrm{ABCD}$ protocol [29], where $A$ stands for the evergreen Asynergy in RWMA, $B$ for $B$-lines, $C$ for Contractile reserve of the left ventricle, and $D$ for Doppler-based CFVR on left anterior descending artery [30].

The present review focuses on force-based imaging of LVCR. It has been well known for decades that contractile reserve of the left ventricle (LV) is an established prognostic predictor. Force is a new parameter but it has old and deep roots in experimental physiology and cardiac imaging, with a variety of different approaches, from nuclear cardiology (myocardial perfusion imaging and radionuclide ventriculography) to echocardiography itself. Force is observed from different perspectives in various subspecialties of cardiology: it is a reduction of the slope in the pressure-volume loop for the cardiac physiologist and invasive cardiologist [31]; a transient ischaemic LV dilation during stress for the cardiac imager [32]; and a drop in systolic blood pressure (SBP) for the clinical cardiologist [33]. All of the above have been independently associated with poor outcome. Nowadays, the parameters of reduced slope of end-systolic pressure (ESP) or end-systolic volume (ESV) curve, transient ischaemic dilation of the LV, and SBP drop during stress converge conceptually, methodologically, and clinically in the systematic use of force-based LVCR during SE (Fig. 1).

\section{THE PHYSIOLOGICAL BASIS OF FORCE}

Ejection fraction (EF) is notoriously dependent on heart rate, as well as on preload and afterload changes [34]. EF values can remain within reference range even after the point of irreversible LV damage has been reached in conditions reducing the LV afterload, such as mitral regurgitation. It is also well established, thanks to the pioneering work of Suga and Sagawa [31] published in the 1970s, that a conceptually immaculate insight into LV contractility can be gained with the pressure-volume loops. During a positive inotropic intervention, the pressure-volume loop reflects a smaller ESV and a higher ESP. In particular, the slope of the pressure-volume loop at end-systole after different loading manipulations and at increasing heart rate identifies the true elastance or contractility value of the individual patient [31]. An increased stimulation rate increases the force of contraction: the molecular explanation is that there is a repetitive $\mathrm{Ca}^{++}$influx with each depolarisation, which leads to an accumulation of cytosolic calcium responsible for $\mathrm{Ca}^{++}$-induced calcium release. As the heart fails, there is a change in the gene expression from the normal adult pattern to that of foetal life, with an inversion of the normal positive slope of the force-frequency relation: 
the processes of systolic calcium release and diastolic calcium reuptake are slower at the basal state, and instead of accelerating with increasing heart rate, they slow down. The increased frequency accelerates $\mathrm{Ca}^{++}$inflow in systole and reuptake from sarcoplasmic reticulum in diastole and justifies the steep force-frequency relationship in healthy hearts or the biphasic-flat relationship in failing hearts. However, this approach requires long, risky, costly, and clinically unrealistic study in the cardiac catheterisation laboratories. The technique remained strictly confined to the academic context for decades [35-37]. Nevertheless, in selected populations submitted to laborious studies, the force evaluated with invasive methods clearly separated hypertrophic cardiomyopathy patients with steep force-frequency relationship and good prognosis from those with flat or biphasic relationship and bad prognosis [38]. This elegant pathophysiological concept was never applied in a clinically realistic non-invasive environment.

\section{THE CLINICAL BASIS OF FORCE:}

\section{LESSONS FROM CUFF SPHYGMOMANOMETERS}

From the very same definition of force, any decrease in SBP during stress is accompanied by a decrease in force reserve (Fig. 2). The normal response to graded exercise is a progressive increase in SBP with no change in diastolic blood pressure. In patients with known or suspected CAD, an increase in SBP is associated with a better prognosis compared with hypotension or a lack of response to dynamic exercise. In fact, exercise-induced hypotension is an established marker of existing and probably severe cardiovascular disease with associated poor prognosis [33, 39]. A reduced SBP is a co-determinant of a decreased value of force. In theory, not only the reduced but also supernormal force values might be "too good to be normal," especially in view of the recognised fact that some diseases (such as hypertrophic cardiomyopathy) may have a transient phase of abnormal compensatory hyperfunction before entering the clinical phase of overt reduction of LVCR and subsequent resting dysfunction [40].

The adverse prognostic meaning of stress-induced hypotension has also been shown for pharmacological stress, although the evidence is clearly much more limited than with regard to exercise. During dobutamine stress a substantial SBP drop (> $20 \mathrm{mmHg}$ during stress compared to resting values) occurs in $15 \%$ of patients and carries an adverse prognostic meaning, as shown in 3381 patients with known or suspected CAD [41] and in 300 patients undergoing major vascular and thoracic non-cardiac surgery [42]. This prognostic value is additive to that of the RWMA presence. During dipyridamole imaging, profound hypotension (stress SBP $<90 \mathrm{mmHg}$ ) was reported in $2.5 \%$ of patients during oral (300 mg) dipyridamole stress and was associated with a fivefold increase in the frequency of severe resting LV dysfunction (19\% vs. $4 \%$ ) [43]. During adenosine or dipyridamole positron emission tomography perfusion imaging, patients with a higher drop in

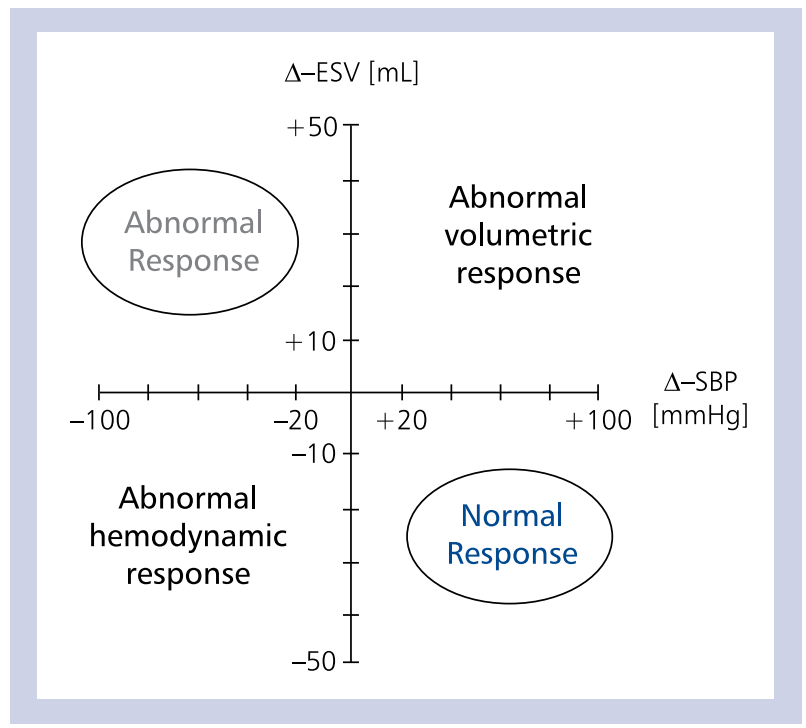

Figure 2. The relationship between force, systolic blood pressure (SBP), and end-systolic volume (ESV): the four quadrants of possible responses during stress. The $x$-axis shows stress-rest variations in SBP; the $y$-axis shows stress-rest variations in ESV

SBP during stress showed a higher risk of death, although the prognostic value did not add significantly to the information provided by resting SBP and inducible ischaemia [44, 45]

\section{LEFT VENTRICULAR DILATION DURING STRESS: THE NUCLEAR CARDIOLOGY EXPERIENCE}

The force is the ratio of SBP and LV ESV. LV dilation has been utilised for over 40 years in cardiac imaging, particularly in myocardial perfusion imaging, as an important variable associated with poor prognosis during physical and pharmacological stress [46]. Transient ischaemic dilation is reflected by an increase in cavity size on post-stress images compared to resting images. In fact, LV dilation is an old but still valid marker of coronary anatomic and prognostic severity, as recently reemphasised in a meta-analysis on 2037 patients from 13 studies ranging from the early 1970s to the present time [47]. Across studies, the annualised rate of cardiac death or myocardial infarction ranged from $0.2 \%$ to $1 \%$ in patients without dilation, from $2 \%$ to $5 \%$ in those with dilation and normal perfusion, and from $5 \%$ to $6 \%$ in those with LV dilation and ischaemia, CAD, or diabetes [47].

\section{LEFT VENTRICULAR DILATION DURING STRESS: THE ECHOCARDIOGRAPHIC EXPERIENCE}

On resting transthoracic echocardiography it is well established that regardless of the EF value, the larger the ESV, the poorer the prognosis, as shown with invasive ventriculographic and noninvasive echocardiographic techniques [48, 49]. The same pattern applies during stress. Thurakhia et al. [50] evaluated 1024 patients enrolled in the prospective Heart 
and Soul study with treadmill SE and found that ESV dilation (stress $>$ rest) was the only significant predictor of mortality (hazard ratio 2.1, 95\% confidence interval 1.43, p < 0.001), even after adjustment for RWMA. ESV was measured with the biplane method of discs and the assessment could be completed in all patients. Patients with ESV dilation also showed lower peak values of SBP [50]. Similar findings on the prognostic impact of LV dilation were obtained in other studies with exercise or pharmacological stress with dobutamine or dipyridamole [51-55]. These studies consistently showed that the prognostic value of LV dilation, assessed with EDV and/ or end-diastolic volume changes, increased the risk stratification value of RWMA and EF, and was associated with anatomically and functionally more severe forms of CAD.

\section{SIMPLIFICATION OF THE CONCEPT OF FORCE}

From a pragmatic point of view, it is necessary to have simple concepts and simple measurements before a successful dissemination of any method in daily practice. Very complex and expensive technologies tend to perform very well in initial efficacy studies but fail to gain prime time in the real world populated by real patients, real doctors, and real problems [56]. SE makes no exception to this "simplify for success" rule.

For the assessment of RWMA, several generations of quantitative methods have been proposed over the years, and although they were very successful in journals, they were never adopted in practice: they were too time consuming, poorly feasible in unselected patients, and dependent on expensive software. The diagnosis today lies — as it did 40 years ago — in careful evaluation by trained readers, because the capability of the human eye to integrate space and time is difficult to match, let alone to surpass.

With regard to $\mathrm{B}$-lines, the most validated and exhaustive scan protocol was the 28-site scan of the antero-lateral chest, which can be performed in only $3 \mathrm{~min}$ and requires $1 \mathrm{~min}$ for reporting in pre-defined forms. The Stress Echo 2020 network accepted in principle the idea to include the assessment of B-lines in the core SE protocol, but only $5 \%$ of centres performed it in their initially recruited cases because it was deemed too time-consuming. Based on this feedback, different scanning protocols were tested and the four-site simplified scan was identified as a reasonable trade-off between accuracy and simplicity [57]. The acceptance rate of this method in the participating centres rose to $100 \%$, and within a few months it was possible to collect stress lung ultrasound data from thousands of patients.

In terms of CFVR assessment, there was a discussion as to whether dual or even triple imaging of the coronary arteries had to be included in the protocol. There is no doubt that double imaging is feasible and informative [58], but once again the issues were time and feasibility. The acceptance rates for imaging of particular arteries were $95 \%$ for the left anterior descending artery (LAD) (duration $<3 \mathrm{~min}$ ), 58\% for the right coronary artery (duration $<5 \mathrm{~min}$ ), and 65\% for the left circumflex artery (duration $<10 \mathrm{~min}$ ). Consequently, only LAD flow imaging was incorporated in the quadruple $A B C D$ protocol, with an extraordinary success rate, and in two years over 4000 patients could be recruited with dual imaging (RWMA and CFVR of LAD).

Also for LVCR the inclusion of the parameter in the dissemination study led to a necessary simplification of its math and semantics. A first attempt to incorporate the assessment of LVCR in the SE protocol was made in 1984 by Ginzton et al. [59], who suggested using cuff sphygmomanometer data as a proxy for ESP and performing echocardiography to assess LV volumes. This innovative approach remained, however, isolated in the literature, apparently abandoned even by those who had originally proposed it. The ESP/ESV ratio was initially called elastance, which was the name used by Suga and Sagawa [31] in their pioneering experimental work. The term force is now preferred, as it is much more familiar to clinical cardiologists, who are inclined to think that a better heart is also a stronger heart. In most studies force is calculated using ESP, which means that peak systolic pressure is multiplied by a correction coefficient of 0.9 . It is common knowledge that peak systolic pressure is higher than ESP, but this correction factor was not introduced in the Stress Echo 2020 study. In the clinical arena, every avoidable computational step is a source of error. We are interested in the relative (stress compared to rest) variation, and therefore any error averages out the results. We therefore deleted the correction factor of 0.9 adopted in previous studies, and we included the raw cuff sphygmomanometer data of SBP in the calculation of force (Table 1).

Further simplification consisted in excluding normalisation for body mass index (BMI) from the formula. In other studies, BMI was included in the assessment of LVCR, but the same values appeared in the numerator (force at peak stress) and in the denominator (force at rest). In the effectiveness studies, values are expressed as raw force (SBP/ESV).

The focus of LVCR is the ratio of peak stress/rest values, and therefore the resulting parameter has several advantages. It is dimensionless and does not need to be expressed in a complex unit of measurement, in contrast to elastance $\left(\mathrm{mmHg} / \mathrm{mL} / \mathrm{m}^{2}\right)$. It takes a range of values very familiar to the cardiologist because normal values for exercise and dobutamine stress are $>2.0$ (as in the case of CFVR), with a titration of abnormal values also mirroring those of CFVR, indicating mild (1.7-2.0), moderate (1.4-1.7) and severe $(<1.4)$ abnormalities.

The issue of feasibility was also important. The assessment of ESV with the method of discs requires biplane views, which are of good quality in most but not all patients. Simpler methods are less accurate for absolute measurements but equally accurate for the evaluation of relative changes, and therefore - when the Simpson method is not feasible - an apical single-plane or even the linear Teichholz method can 
Table 1. Force measurement in efficacy vs. effectiveness studies

\begin{tabular}{lcc} 
& Standard & Simplified \\
Name & Elastance & Force \\
Units & $\mathrm{mmHg} / \mathrm{mL} / \mathrm{m}^{2}$ & None (dimensionless) \\
End-systolic pressure & Peak SBP $\times 0.9$ & SBP \\
End-systolic volume & Simpson biplane method & Area-length or linear method \\
Measurements & All stress steps & Rest and peak stress \\
Analysis time & Minutes & Seconds \\
\hline
\end{tabular}

SBP — systolic blood pressure

be employed. This increases the feasibility to almost 100\% and allows dual imaging (RWMA and LVCR) in virtually all patients [60].

Originally, the evaluation of elastance in the SE lab included measurement in all steps of stress. This was the best way to assess the upsloping vs. flat vs. biphasic response. However, from the prognostic viewpoint the flat and the biphasic patterns have the same meaning; they both generate blunted values of peak force and, therefore, reduced LVCR value. This method was, however, further simplified, so that only rest and peak stress measurements were performed.

Thanks to all these simplifications and reasonable approximations, force and LVCR could be measured in all patients during all types of stress, without an increase in the dedicated imaging time.

\section{FORCE IN STRESS ECHO LAB: HOW TO DO IT}

The force is measured as the ratio of ESP (by cuff sphygmomanometer) to ESV (by two-dimensional [2D] echocardiography). ESV is calculated from apical biplane views or - if one of the views is of poor quality — with apical single-plane view. When the apical views are not of sufficient quality, the linear measurement from parasternal view is accurate in assessing the relative (rest-stress) changes, with accuracy comparable to that of apical biplane method and higher feasibility with shorter analysis time [60]. 2D echocardiography is a relatively precise tool in the evaluation of ESV, with $>90 \%$ of measurements remaining within $10 \%$ difference, and the inter-observer variability of ESV is substantially lower than that of end-diastolic volume [61]. LVCR is the peak stress/rest ratio of left ventricular force. LVCR values during dobutamine or exercise stress may range to indicate normal $(>2.0)$, mild (1.5-2.0), moderate (1.01-1.49), or severe $(\leq 1.0)$ dysfunction. For vasodilators, normal values are lower (abnormal < 1.1) [59].

From the technical viewpoint, force can be assessed and analysed in almost all patients. Image acquisition involves the same $2 \mathrm{D}$ views necessary for the evaluation of RWMA, without additional dedicated imaging. Volume measurement requires around $60 \mathrm{~s}$ (and even less in the case of linear measurements using Teichholz formula). The simple combination of LVCR and RWMA evaluation allows to identify four distinct response

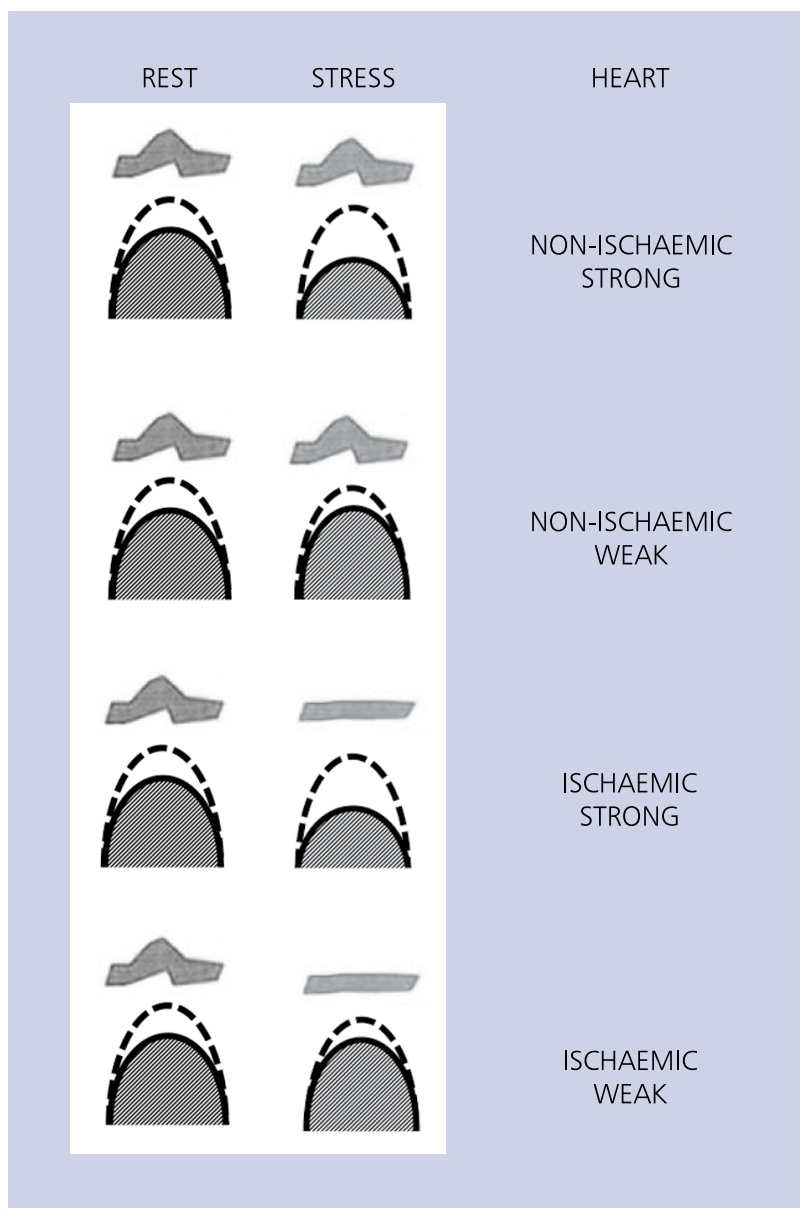

Figure 3. The force and regional wall motion response during stress

patterns: non-ischaemic and strong, non-ischaemic and weak, ischaemic and strong, as well as ischaemic and weak (Fig. 3).

\section{CLINICAL AND PROGNOSTIC VALUE OF FORCE}

Left ventricular contractile reserve is highly feasible during all forms of stress: exercise [62], pacing [63], dobutamine [64], and dipyridamole [65]. In patients with stable angina and normal resting left ventricular function, LVCR reduction during dipyridamole stress showed $86 \%$ sensitivity and $87 \%$ 
Risk stratification beyond regional wall motion abnormalities

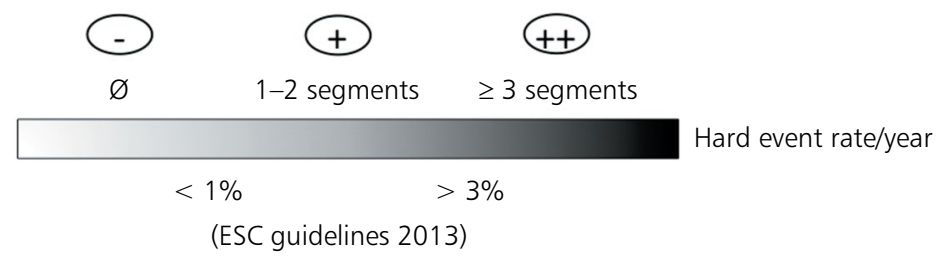

LVCR $>2.0$ (> 1.1 for vasodilator stress)

$\operatorname{LVCR} \leq 2.0(<1.1$ for vasodilator stress $)$

Non-ischaemic strong heart Non-ischaemic weak heart Ischaemic strong heart Ischaemic weak heart

Figure 4. Risk stratification with stress echocardiography. Upper row: stratification based only on the presence of regional wall motion abnormalities (RWMA), endorsed by current guidelines of the European Society of Cardiology (ESC) [3]. Lower row: stratification obtained by dual-imaging, based on the presence of RWMA supplemented with measurement of left ventricular contractile reserve (LVCR); from green for lowest to red for highest risk. The dynamic range of risk stratification can be further expanded by adding B-lines and coronary flow velocity reserve (quadruple imaging)

specificity for the detection of angiographically assessed CAD [66]. In the absence of RWMA, an impaired LVCR is more often present with underlying critical CAD and/or myocardial scar in brain-dead marginal heart donors who underwent autopsy verification after stress [65]. In terms of the prognostic strength, LVCR reduction outperforms the presence of RWMA as well as stress-rest variations in wall motion score index and $E F$, in predicting adverse events including death $[67,68]$. In diabetic patients with no RWMA, the annual hard event rate is threefold higher in persons with abnormal compared to those with normal LVCR [69]. The risk stratification capability of RWMA increases when it is supplemented by an evaluation of LVCR, because an ischaemic response is less malignant in the presence of a strong heart with a preserved global LVCR, and a non-ischaemic response is less benign in the presence of a weak heart with reduced global LVCR (Fig. 4).

\section{THE FUTURE OF FORCE}

Force, as it stands now, is a simple, time-efficient, accurate, and informative parameter. However, all those characteristics can be further improved. Innovations are helping to target the measurement of SBP and ESV and the extension of the concept of force to the right ventricle (RV).

At present, SBP is measured every minute by cuff sphygmomanometer. Peak and true ESPs can be continuously evaluated with a finger tonometer or applanation tonometry on the carotid or brachial artery, and the signal can be easily fed into the echocardiographic machine for real-time, continuous, operator-independent assessment of SBP. A tonometric pulse wave recording is well-tolerated, reproducible, fast, and noninvasive. Its main limitation is the inability to provide absolute values of arterial blood pressure; therefore, a calibration with brachial artery pressure is always required [70].

End-systolic volume is currently efficiently measured by $2 \mathrm{D}$ echocardiography, but real-time three-dimensional (RT3D) echocardiography is obviously more accurate and less time-consuming in the acquisition phase [71]. The volumes can be combined with pressure signal, and a second-generation, operator-independent force is obtained and possibly displayed in real time on the echo monitor.

At present, force is systematically measured for the evaluation of LVCR. However, the same rationale of using force applies to the RV. Well-established indices of RV performance that have been used for the last four decades. These include linear indices based on M-mode (tricuspid annular plane systolic excursion), or those based on 2D (fractional area change in \%) or 3D echocardiography (EF). All of them have limitations because they are heavily load-dependent. Experimentally, the concept of force has been successfully applied to the RV [72], but non-invasive clinical applications have had limited success, mainly because the tricuspid regurgitant velocity (TRV) jet necessary to derive systolic pulmonary artery pressure (SPAP) has a low feasibility at rest and even lower during stress, especially in patients with normal pulmonary haemodynamics (without TRV signal), even after saline echo-contrast injection. However, the feasibility problems have recently been circumvented with the TRACT (Tricuspid Regurgitant velocity + ACceleration Time) protocol: when TRV is absent or unreadable, the systolic pulmonary flow acceleration time is an excellent surrogate with a success rate close to $100 \%$ [73]. This way the RV force (SPAP/ESV) can be obtained in all patients. ESV is usually obtained from the 
monoplane Simpson method applied to the RV imaged from the apical four-chamber view. As with the LV, the estimation of ESV is more reliable and faster with the RT3D approach, which in recent years has gained clinical plausibility thanks to highly improved spatial and temporal resolution and a more ergonomic, relatively small transducer size and footprint [74].

\section{CONCLUSIONS}

With the addition of force to RWMA, SE separates strong hearts with normal LVCR from weak hearts with blunted LVCR. The new force-based parameter needs minimal extra time for imaging and analysis but yields multiple potential benefits. It increases SE versatility and objectivity as well as the positivity rate, broadens the domain of application from CAD to heart failure patients, and improves the risk-stratification potential. The assessment of force and RWMA presence can be also combined with the evaluation of B-lines and CFVR in the full, quadruple-imaging $\mathrm{ABCD}$ core protocol.

Large-scale effectiveness studies with IQ-SE are now underway with the Stress Echo 2020 project, and they will hopefully provide the evidence needed for the inclusion of force into the recommended standard of SE [75]. In the coming years the use of force may become simpler, faster, and more accurate, with radial or carotid applanation tonometry for SBP measurement and RT3D echocardiography for the evaluation of ESV. Force could also be adapted to assess RV performance, with SPAP measured with tricuspid regurgitant jet velocity or acceleration time of systolic forward pulmonary flow velocity. Cardiologists and echocardiographers, upon becoming increasingly familiar with the concept of force, may repeat the mantra of the Star Wars saga: may the force be with you!

\section{Acknowledgements}

Technical assistance of Michele De Nes, an information systems technician at the Pisa Institute of Clinical Physiology of National Research Council, was highly appreciated.

Availability of data and materials: Data will be made available by the Authors upon reasonable request.

Funding: The study was made possible by The Ageing Project funding of the Italian National Research Council (P001328 to Clara Carpeggiani and E.P.).

\section{Conflict of interest: none declared}

\section{References}

1. Picano E. Stress echocardiography. From pathophysiological toy to diagnostic tool. Circulation. 1992; 85(4): 1604-1612, indexed in Pubmed: 1555297.

2. Pellikka PA, Nagueh SF, Elhendy AA, et al. American Society of Echocardiography recommendations for performance, interpretation, and application of stress echocardiography. J Am Soc Echocardiogr. 2007; 20(9): 1021-1041, doi: 10.1016/j. echo.2007.07.003, indexed in Pubmed: 17765820.
3. Sicari R, Nihoyannopoulos P, Evangelista A, et al. European Association of Echocardiography. Stress echocardiography expert consensus statement: European Association of Echocardiography (EAE) (a registered branch of the ESC). Eur J Echocardiogr. 2008; 9(4): 415-437, doi: 10.1093/ejechocard/jen175, indexed in Pubmed: 18579481.

4. Picano E, Pellikka PA. Stress echo applications beyond coronary artery disease. Eur Heart J. 2014; 35(16): 1033-1040, doi: 10.1093/eurheartj/eht350, indexed in Pubmed: 24126880.

5. Lancellotti P, Pellikka PA, Budts W, et al. The clinical use of stress echocardiography in non-ischaemic heart disease: recommendations from the European Association of Cardiovascular Imaging and the American Society of Echocardiography. Eur Heart J Cardiovasc Imaging. 2016; 17(11): 1191-1229, doi: 10.1093/ehjci/jew190, indexed in Pubmed: 27880640.

6. Picano E. Stress echocardiography: a historical perspective. Am J Med. 2003; 114(2): 126-130, doi: 10.1016/s00029343(02)01427-4.

7. Picano E. Sustainability of medical imaging. BMJ. 2004; 328(7439): 578-580, doi: 10.1136/bmj.328.7439.578, indexed in Pubmed: 15001510.

8. Picano E. Informed consent and communication of risk from radiological and nuclear medicine examinations: how to escape from a communication inferno. BMJ. 2004; 329(7470): 849-851, doi: 10.1136/bmj.329.7470.849, indexed in Pubmed: 15472270.

9. Marwick TH, Buonocore J. Environmental impact of cardiac imaging tests for the diagnosis of coronary artery disease. Heart. 2011; 97(14): 1128-1131, doi: 10.1136/hrt.2011.227884, indexed in Pubmed: 21685481.

10. Picano E, Vañó E, Rehani MM, et al. The appropriate and justified use of medical radiation in cardiovascular imaging: a position document of the ESC Associations of Cardiovascular Imaging, Percutaneous Cardiovascular Interventions and Electrophysiology. Eur Heart J. 2014; 35(10): 665-672, doi: 10.1093/eurheartj/eht394, indexed in Pubmed: 24401558.

11. Fonseca R, Otahal P, Wiggins N, et al. Growth and geographical variation in the use of cardiac imaging in Australia. Intern Med J. 2015; 45(11): 1115-1127, doi: 10.1111/imj.12867, indexed in Pubmed: 26247783.

12. Jouni H, Askew JW, Crusan DJ, et al. Temporal trends of single-photon emission computed tomography myocardial perfusion imaging in patients without prior coronary artery disease: A 22-year experience at a tertiary academic medical center. Am Heart J. 2016; 176: 127-133, doi: 10.1016/j.ahj.2016.03.014, indexed in Pubmed: 27264231.

13. Bouzas-Mosquera A, Peteiro J, Broullón FJ, et al. Temporal changes in the use and results of exercise echocardiography. Eur Heart J Cardiovasc Imaging. 2015; 16(11): 1207-1212, doi: 10.1093/ehjci/jev068, indexed in Pubmed: 25851319.

14. Carpeggiani C, Landi P, Michelassi C, et al. The declining frequency of inducible myocardial ischemia during stress echocardiography over 27 consecutive years (1983-2009). Int J Cardiol. 2016; 224: 57-61, doi: 10.1016/j.ijcard.2016.08.313, indexed in Pubmed: 27611918.

15. Cortigiani L, Ramirez P, Coltelli M, et al. Drop-off in positivity rate of stress echocardiography based on regional wall motion abnormalities over the last three decades. Int J Cardiovasc Imaging. 2018; 20: 1501-1503, doi: 10.1007/s10554-018-1501-3, indexed in Pubmed: 30460582.

16. Cortigiani L, Bigi R, Bovenzi F, et al. Prognostic implication of appropriateness criteria for pharmacologic stress echocardiography performed in an outpatient clinic. Circ Cardiovasc Imaging. 2012; 5(3): 298-305, doi: 10.1161/CIRCIMAGING.111.971242, indexed in Pubmed: 22467675.

17. Smulders MW, Jaarsma C, Nelemans PJ, et al. Comparison of the prognostic value of negative non-invasive cardiac investigations in patients with suspected or known coronary artery 
disease-a meta-analysis. Eur Heart J Cardiovasc Imaging. 2017; 18(9): 980-987, doi: 10.1093/ehjci/jex014, indexed in Pubmed: 28329376.

18. Picano E, Severi S, Michelassi C, et al. Prognostic importance of dipyridamole-echocardiography test in coronary artery disease. Circulation. 1989; 80(3): 450-457, indexed in Pubmed: 2766502.

19. Cortigiani L, Paolini EA, Nannini E. Dipyridamole stress echocardiography for risk stratification in hypertensive patients with chest pain. Circulation. 1998; 98(25): 2855-2859, indexed in Pubmed: 9860787.

20. Severi S, Picano E, Michelassi C, et al. Diagnostic and prognostic value of dipyridamole echocardiography in patients with suspected coronary artery disease. Comparison with exercise electrocardiography. Circulation. 1994; 89(3): 1160-1173, indexed in Pubmed: 8124803.

21. Carpeggiani C, Landi P, Michelassi C, et al. Stress echo positivity predicts cancer death. J Am Heart Assoc. 2017; 6(pii:e007104).

22. Cortigiani L, Carpeggiani C, Sicari R, et al. Simple six-item clinical score improves risk prediction capability of stress echocardiography. Heart. 2018; 104(9): 760-766, doi: 10.1136/heartjnl-2017-312122, indexed in Pubmed: 29032362.

23. Picano E, Scali MC. Stress echocardiography, carotid arteries, and more: its versatility for our imaging times. JACC Cardiovasc Imaging. 2018; 11(2 Pt 1): 181-183, doi: 10.1016/j.jcmg.2017.01.023, indexed in Pubmed: 28412415.

24. Picano E, Scali MC, Ciampi Q, et al. Lung ultrasound for the cardiologist. JACC Cardiovasc Imaging. 2018; 11(11): 1692-1705, doi: 10.1016/j.jcmg.2018.06.023, indexed in Pubmed: 30409330.

25. Scali MC, Cortigiani L, Simionuc A, et al. Exercise-induced B-lines identify worse functional and prognostic stage in heart failure patients with depressed left ventricular ejection fraction. Eur J Heart Fail. 2017; 19(11): 1468-1478, doi: 10.1002/ejhf.776, indexed in Pubmed: 28198075.

26. Scali MC, Zagatina A, Ciampi Q, et al. The functional meaning of b-profile during stress lung ultrasound. JACC Cardiovasc Imaging. 2018 [Epub ahead of print], doi: 10.1016/j.jcmg.2018.10.017, indexed in Pubmed: 30553657.

27. Bombardini $\mathrm{T}$, Zoppè $\mathrm{M}$, Ciampi $\mathrm{Q}$, et al. Myocardial contractility in the stress echo lab: from pathophysiological toy to clinical tool. Cardiovasc Ultrasound. 2013; 11: 41, doi: 10.1186/1476-7120-1141, indexed in Pubmed: 24246005.

28. Cortigiani L, Rigo F, Gherardi S, et al. Coronary flow reserve during dipyridamole stress echocardiography predicts mortality. JACC Cardiovasc Imaging. 2012; 5(11): 1079-1085, doi: 10.1016/j. jcmg.2012.08.007, indexed in Pubmed: 23153906.

29. Picano E, Morrone D, Scali MC, et al. Integrated quadruple stress echocardiography. 2018; [Epub ahead of print], doi: 10.23736/S0026-4725.18.0469-1-1.

30. Picano E, Ciampi Q, Wierzbowska-Drabik K, et al. The new clinical standard of integrated quadruple stress echocardiography with ABCD protocol. Cardiovasc Ultrasound. 2018; 16(1): 22, doi: 10.1186/s12947-018-0141-z, indexed in Pubmed: 30285774.

31. Suga H, Sagawa K, Shoukas AA. Load independence of the instantaneous pressure-volume ratio of the canine left ventricle and effects of epinephrine and heart rate on the ratio. Circ Res. 1973; 32(3): 314-322, indexed in Pubmed: 4691336.

32. Taillefer R. Transient dilation of the left ventricular cavity observed during myocardial perfusion imaging: What is its incremental diagnostic value. J Nucl Cardiol. 2018; 25(2): 593-595, doi: 10.1007/s12350-016-0701-2, indexed in Pubmed: 27804068.

33. Fletcher GF, Ades PA, Kligfield P, et al. Exercise standards for testing and training: a scientific statement from the American Heart Association. Circulation. 2013; 128(8): 873-934, doi: 10.1161/CIR.0b013e31829b5b44, indexed in Pubmed: 23877260.

34. Cikes M, Solomon SD. Beyond ejection fraction: an integrative approach for assessment of cardiac structure and function in heart failure. Eur Heart J. 2016; 37(21): 1642-1650, doi: 10.1093/eurhearti/ehv510, indexed in Pubmed: 26417058.

35. Bombardini T. Myocardial contractility in the echo lab: molecular, cellular and pathophysiological basis. Cardiovasc Ultrasound. 2005; 3: 27, doi: 10.1186/1476-7120-3-27, indexed in Pubmed: 16150150.

36. Liu CP, Ting CT, Lawrence W, et al. Diminished contractile response to increased heart rate in intact human left ventricular hypertrophy. Systolic versus diastolic determinants. Circulation. 1993; 88(4 Pt 1): 1893-1906, indexed in Pubmed: 8403335.

37. Yokoyama Y, Cooper DK, Sasaki H, et al. Donor-heart evaluation by monitoring the left ventricular pressure-volume relationship: clinical observations. J Heart Lung Transplant. 1992; 11(4 Pt 1): 685-692, indexed in Pubmed: 1498132.

38. Morimoto R, Okumura T, Bando YK, et al. Biphasic force-frequency relation predicts primary cardiac events in patients with hypertrophic cardiomyopathy. Circ J. 2017; 81(3): 368-375, doi: 10.1253/circj.CJ-16-1007, indexed in Pubmed: 28025461.

39. O'Neal WT, Qureshi WT, Blaha MJ, et al. Systolic blood pressure response during exercise stress testing: the henry ford exercise testing (FIT) project. J Am Heart Assoc. 2015; 4(5), doi: 10.1161/JAHA.115.002050, indexed in Pubmed: 25953655.

40. Green EM, Wakimoto H, Anderson RL, et al. A small-molecule inhibitor of sarcomere contractility suppresses hypertrophic cardiomyopathy in mice. Science. 2016; 351(6273): 617-621, doi: 10.1126/science.aad3456, indexed in Pubmed: 26912705.

41. Dunkelgrun M, Hoeks SE, Elhendy A, et al. Significance of hypotensive response during dobutamine stress echocardiography. Int J Cardiol. 2008; 125(3): 358-363, doi: 10.1016/j. ijcard.2007.02.058, indexed in Pubmed: 17466395.

42. Day SM, Younger JG, Karavite D, et al. Usefulness of hypotension during dobutamine echocardiography in predicting perioperative cardiac events. Am J Cardiol. 2000; 85(4): 478-483, indexed in Pubmed: 10728954

43. Perper EJ, Segall GM. Safety of dipyridamole-thallium imaging in high risk patients with known or suspected coronary artery disease. J Nucl Med. 1991; 32(11): 2107-2114, indexed in Pubmed: 1941146

44. Witbrodt B, Goyal A, Kelkar AA, et al. Prognostic significance of blood pressure response during vasodilator stress $\mathrm{Rb}-82$ positron emission tomography myocardial perfusion imaging. J Nucl Cardiol. 2017; 24(6): 1966-1975, doi: 10.1007/s12350-016-0569-1, indexed in Pubmed: 27659457.

45. Reyes E, Hage FG. The blood pressure response to vasodilator stress does not provide independent prognostic information. J Nucl Cardiol. 2017; 24(6): 1976-1978, doi: 10.1007/s12350-0160651-8, indexed in Pubmed: 27572924.

46. Bajaj NS, Singh S, Farag A, et al. The prognostic value of non-perfusion variables obtained during vasodilator stress myocardial perfusion imaging. J Nucl Cardiol. 2016; 23(3): 390-413, doi: 10.1007/s12350-016-0441-3, indexed in Pubmed: 26940574.

47. Alama M, Labos C, Emery H, et al. Diagnostic and prognostic significance of transient ischemic dilation (TID) in myocardial perfusion imaging: A systematic review and meta-analysis. J Nucl Cardiol. 2018; 25(3): 724-737, doi: 10.1007/s12350-017-1040-7, indexed in Pubmed: 28948540.

48. Matsuzaki M, Gallagher KP, Kemper WS, et al. Sustained regional dysfunction produced by prolonged coronary stenosis: gradual recovery after reperfusion. Circulation. 1983; 68(1): 170-182, indexed in Pubmed: 6851044.

49. Nicolosi GL, Latini R, Marino P, et al. The prognostic value of predischarge quantitative two-dimensional echocardiographic measurements and the effects of early lisinopril treatment on left ventricular structure and function after acute myocardial infarction in the GISSI-3 Trial. Gruppo Italiano per lo Studio della Sopravvivenza nell'Infarto Miocardico. Eur Heart J. 1996; 17(11): 1646-1656, indexed in Pubmed: 8922912. 
50. Turakhia MP, McManus DD, Whooley MA, et al. Increase in end-systolic volume after exercise independently predicts mortality in patients with coronary heart disease: data from the Heart and Soul Study. Eur Heart J. 2009; 30(20): 2478-2484, doi: 10.1093/eurheartj/ehp270, indexed in Pubmed: 19578167.

51. Coletta C, Galati A, Ricci R, et al. Prognostic value of left ventricular volume response during dobutamine stress echocardiography. Eur Heart J. 1997; 18(10): 1599-1605, indexed in Pubmed: 9347270.

52. Porter TR, Li S, Kricsfeld D, et al. Detection of myocardial perfusion in multiple echocardiographic windows with one intravenous injection of microbubbles using transient response second harmonic imaging. J Am Coll Cardiol. 1997; 29(4): 791-799, indexed in Pubmed: 9091526.

53. Jacobs LD, Salgo IS, Goonewardena S, et al. Rapid online quantification of left ventricular volume from real-time three-dimensional echocardiographic data. Eur Heart J. 2006; 27(4): 460-468, doi: 10.1093/eurheartj/ehi666, indexed in Pubmed: 16319085.

54. Yao SS, Shah A, Bangalore S, et al. Transient ischemic left ventricular cavity dilation is a significant predictor of severe and extensive coronary artery disease and adverse outcome in patients undergoing stress echocardiography. J Am Soc Echocardiogr. 2007; 20(4): 352-358, doi: 10.1016/j.echo.2006.09.014, indexed in Pubmed: 17400113.

55. Kataoka A, Scherrer-Crosbie M, Senior R, et al. Transient ischemic dilatation during stress echocardiography: an additional marker of significant myocardial ischemia. Echocardiography. 2016; 33(8): 1202-1208, doi: 10.1111/echo.13222, indexed in Pubmed: 27040889.

56. Cheng SC, Dy T, Feinstein S. Contrast echocardiography: review and future directions. Am J Cardiol. 1998; 81(12): 41-48, doi: 10.1016/s0002-9149(98)00053-8.

57. Scali MC, Zagatina A, Simova I, et al. B-lines with lung ultrasound: the optimal scan technique at Rest and during stress. Ultrasound Med Biol. 2017; 43(11): 2558-2566, doi: 10.1016/j. ultrasmedbio.2017.07.007, indexed in Pubmed: 28865726.

58. Cortigiani L, RigoF, Bovenzi F, et al. The Prognostic Value of Coronary Flow Velocity Reserve in Two Coronary Arteries During Vasodilator Stress Echocardiography. J Am Soc Echocardiogr. 2019; 32(1): 81-91, doi: 10.1016/j.echo.2018.09.002, indexed in Pubmed: 30482637.

59. Ginzton LE, Laks MM, Brizendine M, et al. Noninvasive measurement of the rest and exercise peak systolic pressure/end-systolic volume ratio: a sensitive two-dimensional echocardiographic indicator of left ventricular function. J Am Coll Cardiol. 1984; 4(3): 509-516, indexed in Pubmed: 6470330.

60. Torres MA, Texeira MF, Bellagamba CC, et al. Left ventricular contractile reserve during stress-echo: the value of a simplified approach. EuroEcho-Imaging. 2018; FP Number: P783

61. Crowley AL, Yow E, Barnhart HX, et al. Critical review of current approaches for echocardiographic reproducibility and reliability assessment in clinical research. J Am Soc Echocardiogr. 2016; 29(12): 1144-1154.e7, doi: 10.1016/j.echo.2016.08.006, indexed in Pubmed: 27720558.

62. Bombardini T, Correia M, Cicerone C, et al. Force-frequency relationship in the echocardiography laboratory: a noninvasive assessment of Bowditch Treppe? J Am Soc Echo. 2003; 17(6): 832-841, doi: 10.1016/s0894-7317(03)00221-9.

63. Bombardini T, Agrusta M, Natsvlishvili N, et al. Noninvasive assessment of left ventricular contractility by pacemaker stress echocardiography. Eur J Heart Fail. 2005; 7(2): 173-181, doi: 10.1016/j. ejheart.2004.04.019, indexed in Pubmed: 15701463.
64. Grosu A, Bombardini T, Senni M, et al. End-systolic pressure/volume relationship during dobutamine stress echo: a prognostically useful non-invasive index of left ventricular contractility. Eur Heart J. 2005; 26(22): 2404-2412, doi: 10.1093/eurheartj/ehi444, indexed in Pubmed: 16105848.

65. Leone O, Gherardi S, Targa L, et al. Stress echocardiography as a gatekeeper to donation in aged marginal donor hearts: anatomic and pathologic correlations of abnormal stress echocardiography results. J Heart Lung Transplant. 2009; 28(11): 1141-1149, doi: 10.1016/j.healun.2009.05.029, indexed in Pubmed: 19782600

66. Bombardini T, Gherardi S, Marraccini P, et al. The incremental diagnostic value of coronary flow reserve and left ventricular elastance during high-dose dipyridamole stress echocardiography in patients with normal wall motion at rest. Int J Cardiol. 2013; 168(2): 1683-1684, doi: 10.1016/j.ijcard.2013.03.076, indexed in Pubmed: 23601214.

67. Cortigiani L, Bombardini T, Corbisiero A, et al. The additive prognostic value of end-systolic pressure-volume relation in patients with diabetes mellitus having negative dobutamine stress echocardiography by wall motion criteria. Heart. 2009; 95(17): 1429-1435, doi: 10.1136/hrt.2008.161752, indexed in Pubmed: 19414439 .

68. Matsumoto K, Tanaka H, Onishi A, et al. Bi-ventricular contractile reserve offers an incremental prognostic value for patients with dilated cardiomyopathy. Eur Heart J Cardiovasc Imaging. 2015; 16(11): 1213-1223, doi: 10.1093/ehjci/jev069, indexed in Pubmed: 25851330.

69. Cortigiani L, Huqi A, Ciampi Q, et al. Integration of wall motion, coronary flow velocity, and left ventricular contractile reserve in a single test: prognostic value of vasodilator stress echocardiography in patients with diabetes. J Am Soc Echocardiogr. 2018; 31(6): 692-701, doi: 10.1016/j.echo.2017.11.019, indexed in Pubmed: 29625884.

70. Salvi P, Grillo A, Parati G. Noninvasive estimation of central blood pressure and analysis of pulse waves by applanation tonometry. Hypertens Res. 2015; 38(10): 646-648, doi: 10.1038/hr.2015.78, indexed in Pubmed: 26155750.

71. Berbarie RF, Dib E, Ahmad M. Stress echocardiography using real-time three-dimensional imaging. Echocardiography. 2018; 35(8): 1196-1203, doi: 10.1111/echo.14050, indexed in Pubmed: 30133883.

72. Hsu S, Houston BA, Tampakakis E, et al. Right ventricular functional reserve in pulmonary arterial hypertension. Circulation. 2016; 133(24): 2413-2422, doi: 10.1161/CIRCULATIONAHA.116.022082, indexed in Pubmed: 27169739.

73. Wierzbowska-Drabik K, Picano E, Bossone E, et al. The feasibility and clinical implication of tricuspid regurgitant velocity and pulmonary flow acceleration time for pulmonary pressure assessment during exercise stress echocardiography. Eur Heart J Cardiovasc Imaging. 2019 (in press). jez029.

74. Ostenfeld E, Flachskampf FA. Assessment of right ventricular volumes and ejection fraction by echocardiography: from geometric approximations to realistic shapes. Echo Res Pract. 2015; 2(1): R1-R11, doi: 10.1530/ERP-14-0077, indexed in Pubmed: 26693327.

75. Picano E, Ciampi Q, Citro R, et al. Stress echo 2020: the international stress echo study in ischemic and non-ischemic heart disease. Cardiovasc Ultrasound. 2017; 15(1): 3, doi: 10.1186/s12947016-0092-1, indexed in Pubmed: 28100277.

Cite this article as: Picano E, Bombardini T, Kovačević Preradović T, et al. Left ventricular contractile reserve in stress echocardiography: the bright side of the force. Kardiol Pol. 2019; 77(2): 164-172, doi: 10.5603/KP.a2019.0002. 